\title{
NLOS Identification and Compensation for UWB Ranging Based on Obstruction Classification
}

\author{
Kai Wen, Kegen Yu and Yingbing Li \\ School of Geodesy and Geomatics and Collaborative Innovation Center for Geospatial Technology \\ Wuhan University, Wuhan, China \\ wenkai@whu.edu.cn; kgyu@sgg.whu.edu.cn; ybli@sgg.whu.edu.cn
}

\begin{abstract}
Non-line-of-sight (NLOS) propagation is one of the major barriers to accurate ranging and positioning based on time of arrival (TOA) in the application of an ultra wideband (UWB) system. This paper proposes a new method for NLOS identification and mitigation based on signal characteristic analysis and fuzzy theory. This method neither requires to build a statistical model nor to create and update a training database, so that it can be used conveniently for different application scenarios. Extensive experiments were conducted and the results show that the cumulative distribution function of the ranging error below 0.5 meter is over $90 \%$ when using the proposed mitigation method, while that without using the mitigation method is below $70 \%$. Also, by using the proposed method, the root mean square error (RMSE) of the range measurements is reduced from 0.77 to 0.33 meter. The results demonstrate that this method can effectively identify NLOS and mitigate the NLOS-induced ranging error.
\end{abstract}

Keywords-UWB; NLOS identification; fuzzy theory; error mitigation

\section{INTRODUCTION}

Although UWB technology owns the ability of penetrating obstructions, such as concrete, brick, glass and wood, the penetration leads to that signal is delayed and decayed considerably. As a consequence, in the complex indoor environment where the NLOS propagation occurs, the ranging and thus positioning accuracy based on time measurement is degraded significantly. To mitigate the influence of NLOS and hence improve positioning accuracy, different methods have been proposed in the literature for NLOS identification and error mitigation. In [1] the main NLOS identification techniques are classified into three categories, namely range estimation-based methods, channel statistics-based methods and position estimation-based methods. Meanwhile, NLOS error mitigation techniques are divided into statistics-based methods and direct path estimation-based methods. Channel statistics-based methods for NLOS identification produce better performance compared to other methods [1]. The methods usually use hypothesis testing or classification theory for NLOS identification based on channel impulse response (CIR) characteristics. The main drawback of current statistics-based methods is that these methods are commonly developed under idealized conditions or it is required to build and frequently update a large training database. Reference [2] proposes a non-parametric machine learning technique to perform NLOS identification and mitigation. Since statistical characteristics of LOS and NLOS channels are not required, the method can be used under a common framework. However, extensive work is needed to update the training database in order to ensure accurate identification, leading to increased labor cost and reduced usability.

This paper proposes a new method for NLOS identification and mitigation based on signal characteristic analysis. The main innovations and advantages of the proposed method are: (i) the factors resulting in NLOS propagation in practical applications are categorized as a number of typical representatives; (ii) the influence of NLOS on ranging is considered as a combination of respective effects of different factors; (iii) model modification is not needed once a model is built; (iv) the complexity and time consumption is much smaller compared to other range-based methods. The remainder of the paper is organized as follows. In section II, signal characteristics under NLOS conditions are analyzed. In section III, the proposed method is deduced and described. In section $\mathrm{IV}$, an experiment is conducted to verify the proposed method, and finally, a conclusion is drawn in section $\mathrm{V}$.

\section{SIGNAL CHARACTERISTICS ANALYSIS UNDER NLOS PROPAGATION CONDITIONS}

In this section, waveforms and CIRs in time domain under different propagation conditions are analyzed based on measurements made by P410 RCM. The P410 RCM is a multi-purpose platform based on UWB technology that can be used for peer-to-peer distance measurement, data communication, and CIR measurement. The P410 RCM uses two-way time-of-flight (TW-TOF) to accurately measure the distance between two units [3]. During each range measurement, two types of scan data of the pulse waveform are provided, which are a short scan of 350 points of data and a full scan of 1632 points of data [3]. The scan information can be used to investigate the CIR around the direct path and make conclusions about the range or channel multipath content.

Fig. 1 and Fig. 2 show the received waveforms and corresponding CIRs from six different propagation conditions, namely outdoor LOS, indoor LOS, propagating around a corner of a corridor, penetrating through a wood door, an iron door and a concrete wall. All waveforms are recorded by the same devices. In the case of outdoor LOS, we can find that the recorded waveform is similar with the original waveform (which can be found from: 
http://www.timedomain.com/). While recorded waveforms in other cases change more dramatically. The signal amplitudes are obviously reduced under NLOS propagation conditions, except for the case of penetrating through a wood door, compared to those of LOS propagation conditions. The most serious variation is observed in propagating around a corner and through-the-iron-door cases. The noise level under LOS conditions are low and stable, but it changes significantly under NLOS conditions except for the through-the-wood-door case. This is because the refracted and scattered signals from the surface or edge of obstruction arrive at the receiver with different time delays. Fig. 3 displays the abnormality of waveform induced by penetrating through an iron door. Due to the presence of an iron door, the amplitudes of waveform are reduced and distorted greatly. Fig. 4 shows three received waveforms penetrating through three different iron doors in the same building. It turns out that the received waveforms are very similar when penetrating through the same type of obstructions ${ }^{1}$.

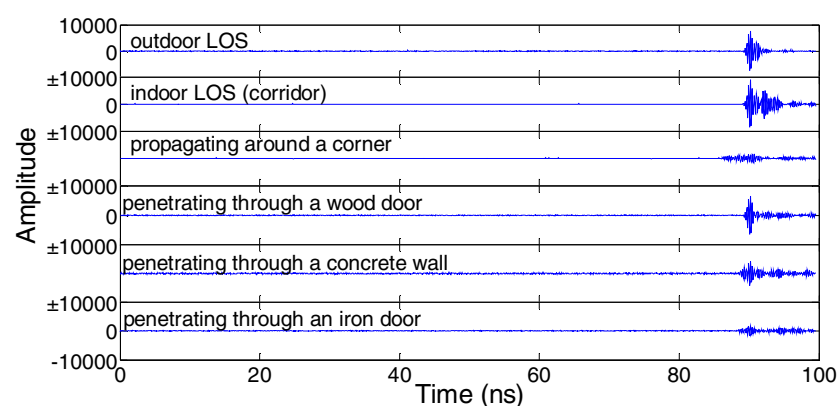

Figure 1. Received waveforms in LOS and NLOS environments ${ }^{2}$.

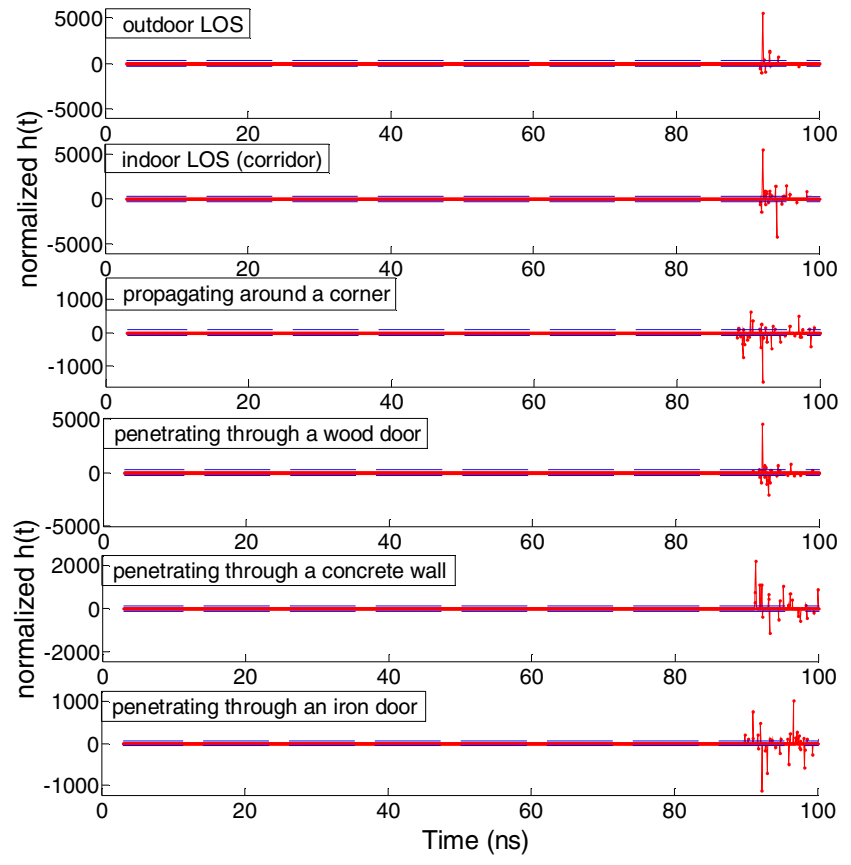

Figure 2. CIRs of received waveforms (the conversion between CIR and waveform is accomplished using CLEAN algorithm, threshold $=25 \mathrm{~dB}$ ).

${ }^{1}$ For a real scenario, the used doors commonly have little difference although they may come from different manufacturers.

2 The amplitude denotes the signal strength relative to that of the first arriving signal.

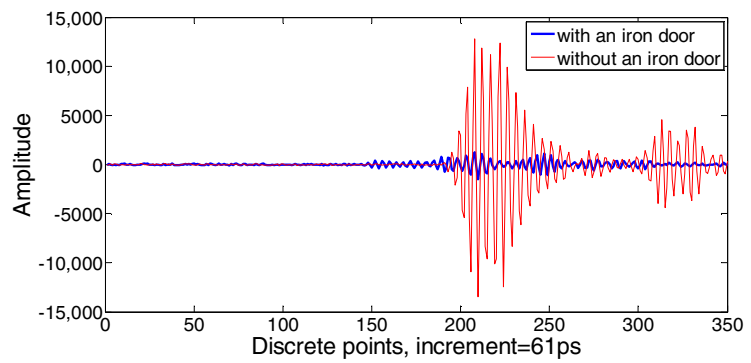

Figure 3. Received waveforms with and without an iron door in the propagation path.

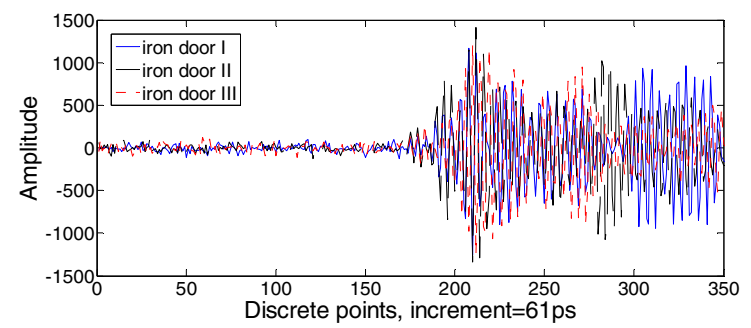

Figure 4. Received waveforms penetrating through three iron doors made by three different manufacturers respectively.

\section{THE PROPOSED METHOD}

\section{A. Overview of the Proposed Method}

NLOS propagation occurs when the signal propagation path is blocked by obstructions. Indoors, the common obstructions include wall, door, furniture, and pedestrian. Although there are differences among ranging errors caused by different obstructions, the fundamental cause leading to ranging errors under various conditions is two-fold, power decay and time delay. Therefore we can broadly classify common factors resulting in NLOS as a number of typical representatives which are penetration through an iron door, a wood door and a concrete wall, propagation around a corner, and interfering from pedestrians. The ranging errors and the CIR caused by each type of obstructions can be collected in advance. Then, the factors causing NLOS can be identified by analyzing the characteristics of a specific CIR, and the corresponding ranging measurements can be corrected using the collected priori ranging errors ${ }^{3}$. Actually NLOS propagation is not introduced by only one factor, but several factors simultaneously. For instance, signal may penetrate through a concrete wall as well as a wood board, or be disturbed by pedestrians and furniture simultaneously. It is inaccurate to treat multiple obstructions as a single one. Hence, for a typical NLOS propagation scenario, it is reasonable to consider that the ranging error results from the total effect of several factors. In the proposed method, a comprehensive evaluation model based on fuzzy theory is used in both NLOS identification and mitigation stages.

\section{B. Fuzzy Comprehensive Evaluation Model}

Fuzzy comprehensive evaluation model consists of three basic elements:

(i) factor set:

\footnotetext{
3 Strictly speaking, the effect of identified factors is only equivalent to that of the true factors since this method cannot cover all factors resulting in NLOS.
} 


$$
X=\left\{x_{1}, x_{2}, \cdots, x_{n}\right\}
$$

where $x_{i}(i=1,2, \cdots, n)$ represents a factor that needs to be considered in making the judgment;

(ii) judgement set:

$$
Y=\left\{y_{1}, y_{2}, \cdots, y_{m}\right\}
$$

where $y_{j}(j=1,2, \cdots, m)$ is a potential judgment;

(iii) fuzzy mapping [4]:

$$
\tilde{f}: X \rightarrow \mathscr{F}(Y), x_{i} \mapsto \tilde{f}\left(x_{i}\right) \underline{=}\left(\begin{array}{llll}
r_{i 1} & r_{i 2} & \cdots & r_{i m}
\end{array}\right),
$$

where $\tilde{f}$ is the fuzzy mapping from $X$ to $Y, \mathscr{F}(Y)$ is the nonempty fuzzy sets of $Y$, and $\left(\begin{array}{llll}r_{i 1} & r_{i 2} & \cdots & r_{i m}\end{array}\right)$ is one row of matrix $\underset{\sim}{R}$, which indicates the fuzzy relation between $X$ and $Y \stackrel{\sim}{\text { as }}$

$$
\underset{\sim}{R}=\left[\begin{array}{cccc}
r_{11} & r_{12} & \cdots & r_{1 m} \\
r_{21} & r_{22} & \cdots & r_{2 m} \\
\cdots & \cdots & \cdots & \cdots \\
r_{n 1} & r_{n 2} & \cdots & r_{n m}
\end{array}\right],
$$

where $r_{i j}(i=1,2, \cdots, n ; j=1,2, \cdots, m)$ represents the probability that judgment belong to $y_{j}$ based on $x_{i}$.

Fuzzy theory argues that it is unnecessary to judge a result either positive or negative. Instead, the judgment can be seen as a fuzzy set of $Y$ as

$$
\underset{\sim}{B}=\left(b_{1}, b_{2}, \cdots, b_{m}\right) \in \mathscr{F}(Y),
$$

where $b_{j}(j=1,2, \cdots, m)$ represents the probability that the judgment belongs to $y_{j}$.

Assuming a fuzzy set of factor set $X$ is

$$
\underset{\sim}{A}=\left(a_{1}, a_{2}, \cdots, a_{n}\right) \in \mathscr{F}(X), \quad \sum_{i=1}^{n} a_{i}=1,
$$

where $a_{i}(i=1,2, \cdots, n)$ represents the degree of influence of factor $x_{i}$ on judgment, and $\mathscr{F}(X)$ is the nonempty fuzzy sets of $X$. The comprehensive evaluation result can be obtained by a fuzzy transformation as in

$$
\underset{\sim}{B}=\underset{\sim}{A} \stackrel{\circ}{\sim}
$$

where " $\circ "$ indicates the operation defined as in

$$
b_{j}=\left(a_{1} \wedge r_{1 j}\right) \vee\left(a_{1} \wedge r_{2 j}\right) \vee \cdots \vee\left(a_{1} \wedge r_{n j}\right),
$$

here, $\wedge$ is the operation of selecting the smaller number, while $\vee$ chooses the larger number.

\section{NLOS Identification and Compensation}

Signal quality is commonly described by metrics of energy of the received signal, maximum amplitude, rise time (RT), RMS delay spread, kurtosis, skewness, and SNR [2], [5]. In our method, SNR, RT, RMS delay spread, kurtosis and skewness are used to identify NLOS based on following reasons: (i) RMS delay spread increases when more multipath components are included in received signal [5], [6]; (ii) signal channel's structure does not change appreciably over short distances, but drastic variation arises when there is an increase in the number of intervening obstructions [6], [7]; (iii) the energy, when signal penetrates through obstructions, will be seriously decayed, but noise increases inversely. These chosen metrics are calculated as in (9)-(13) [2], [5].

(i) Kurtosis:

$$
K=\frac{1}{(n-1) s^{4}} \sum_{i=1}^{n}\left(\left|a_{i}\right|-\bar{a}\right)^{4}
$$

(ii) skewness:

$$
S=\frac{1}{(n-1) s^{3}} \sum_{i=1}^{n}\left(\left|a_{i}\right|-\bar{a}\right)^{3}
$$

(iii) rise time $(\mathrm{RT})$ :

$$
\begin{aligned}
& R T=t_{e}-t_{s}, \\
& \left\{\begin{array}{l}
t_{s}=\min \left\{t:\left|a_{i}\right| \geqslant \alpha \cdot n\right\} \\
t_{e}=\min \left\{t:\left|a_{i}\right| \geqslant \beta \cdot v\right\}
\end{array}\right.
\end{aligned}
$$

(iv) root-mean-square (RMS) delay spread:

$$
\begin{aligned}
& \tau_{r m s}=\sqrt{\left[\sum_{i=1}^{n}\left(t_{i}-\tau_{m}\right)^{2} \cdot a_{i}^{2}\right] / E,} \\
& E=\sum_{i=1}^{n} a_{i}^{2}, \tau_{m}=\sum_{i=1}^{n} t_{i} a_{i}^{2} / E
\end{aligned}
$$

(v) signal-to-noise ratio (SNR):

$$
S N R=20 \cdot \lg (v / n) ;
$$

where $a_{i}$ and $t_{i}$ are the amplitude and the arrival time of the random path in CIR; $v, \bar{a}$ and $s$ are the maximum, mean and standard deviation of amplitudes of all recorded paths in CIR respectively; $n$ is noise level and $\alpha$ and $\beta$ are set as 4 and 0.95 in this model. Following steps are used to identify NLOS and compensate errors.

Firstly, the factor set and determination set are built as

$$
\left\{\begin{array}{l}
X=\left\{x_{\tau}, x_{k}, x_{s}, x_{r}, x_{n}\right\} \\
Y=\left\{y_{i}, y_{w}, y_{c}, y_{p}, y_{o}\right\}
\end{array},\right.
$$

where $x_{\tau}, x_{k}, x_{s}, x_{r}$ and $x_{n}$ respectively represent RMS delay spread, kurtosis, skewness, RT and SNR, and $y_{i}, y_{w}, y_{c}, y_{p}$ and $y_{o}$ respectively represent the five hypothetical typical propagation conditions, namely iron door, wood door, concrete wall, pedestrian, and corner. In a specific application, the elements in $Y$ are chosen according to the representative propagation conditions in the actual environment. Assume that the degree of influence of each factor on judgment is

$$
\underset{\sim}{A}=\left(w_{\tau}, w_{k}, w_{s}, w_{r}, w_{n}\right) .
$$

Then, collect the priori values of CIR characteristics and ranging errors under the five propagation conditions. 
Later, for a specific measured CIR with $x_{0}=\left(\tau_{0}, k_{0}, s_{0}, r_{0}, n_{0}\right)$, the probability that $x_{0}$ belongs to $y_{i}$ denoted by $\mu_{i^{*}}\left(x_{0}\right)$ is calculated as

$$
\begin{aligned}
\mu_{i^{*}}\left(x_{0}\right)= & w_{\tau} \cdot \mu_{i}\left(\tau_{0}\right)+w_{k} \cdot \mu_{i}\left(k_{0}\right) \\
& +w_{s} \cdot \mu_{i}\left(s_{0}\right)+w_{r} \cdot \mu_{i}\left(r_{0}\right)+w_{n} \cdot \mu_{i}\left(n_{0}\right),
\end{aligned}
$$

where $\mu_{i}\left(\tau_{0}\right), \mu_{i}\left(k_{0}\right), \mu_{i}\left(s_{0}\right), \mu_{i}\left(r_{0}\right)$ and $\mu_{i}\left(n_{0}\right)$ denote the probabilities that $x_{0}$ belongs to $y_{i}$ determined by individual factors respectively. Specifically, $\mu_{i}\left(\tau_{0}\right)$ is calculated by

$$
\begin{aligned}
& \mu_{i}\left(\tau_{0}\right)=\frac{1}{p} \sum_{k=1}^{p}\left(1-\left|\frac{\tau_{0}-\tau_{k}}{\tau_{0}}\right|\right), \\
& \text { if }\left|\frac{\tau_{0}-\tau_{k}}{\tau_{0}}\right|>1, \text { then }\left|\frac{\tau_{0}-\tau_{k}}{\tau_{0}}\right|=1,
\end{aligned}
$$

where $\tau_{k}$ stands for the value of RMS delay spread of the $k$ th priori CIR. $\mu_{i}\left(k_{0}\right), \mu_{i}\left(s_{0}\right), \mu_{i}\left(r_{0}\right)$ and $\mu_{i}\left(n_{0}\right)$ are calculated in the same way.

Likewise, the probability that $x_{0}$ belongs to other cases can be obtained as $\mu_{w^{*}}\left(x_{0}\right), \mu_{c^{*}}\left(x_{0}\right), \mu_{p^{*}}\left(x_{0}\right)$ and $\mu_{o^{*}}\left(x_{0}\right)$. By normalization, the comprehensive evaluation result can be obtained as

$$
\begin{aligned}
& B=\left(\frac{\mu_{i^{*}}\left(x_{0}\right)}{\mu}, \frac{\mu_{w^{*}}\left(x_{0}\right)}{\mu}, \frac{\mu_{c^{*}}\left(x_{0}\right)}{\mu}, \frac{\mu_{p^{*}}\left(x_{0}\right)}{\mu}, \frac{\mu_{o^{*}}\left(x_{0}\right)}{\mu}\right), \\
& \mu=\mu_{i^{*}}\left(x_{0}\right)+\mu_{w^{*}}\left(x_{0}\right)+\mu_{c^{*}}\left(x_{0}\right)+\mu_{p^{*}}\left(x_{0}\right)+\mu_{o^{*}}\left(x_{0}\right) .
\end{aligned}
$$

Lastly, range measurements can be corrected by

$$
\hat{L}=L+B \cdot\left(l_{i}, l_{w}, l_{c}, l_{p}, l_{o}\right)^{T}
$$

where $L$ and $\hat{L}$ are the measured and corrected range respectively, and $l_{i}, l_{w}, l_{c}, l_{p}$ and $l_{o}$ are priori errors under the different propagation conditions. Each priori error under a certain propagation condition is determined by averaging multiple ranging errors in different locations ${ }^{4}$. The method is rough but rational because the ranging error is mainly relative with the thickness, material and humidity of the obstruction, and that these factors are nearly identical for the same type of obstructions in a certain environment [8], [9].

\section{EXPERIMENT AND RESULTS}

\section{A. Experimental Setup}

The experiment was conducted on the first floor of the twelfth student lodge within Wuhan University, Wuhan, China. That is a rather complex indoor environment, containing hallway, wall, corridor, toilet, laundry and room in addition to bed and cabinet. The experiment consists of two stages. In the first stage, priori values of signal characteristic and errors under different propagation conditions were collected. In the second stage, nineteen differ-

${ }^{4}$ In order to simplify the model, we assume that UWB signal can penetrate only one wall instead of multiple walls. Actually, signal may penetrate two or even more walls, but the signal power may become very weak so that the measured range could not be used. ent locations under NLOS conditions and five different locations under LOS conditions were selected and data were recorded. All measured ranges are within 10 meters because of being limited by environment. The distribution of test locations is shown in Fig. 5.

In the first stage, we divided propagation conditions into five categories, namely indoor LOS, propagating around a corner, through-the-door, through-the-wall, and interfering by pedestrians, and range measurements were collected at five typical locations for each condition. Each range was measured 100 times repeatedly, hence 100 CIRs and ranging errors were recorded. Based on these recorded CIRs, we calculate the priori values of SNR, RT, kurtosis, skewness and RMS delay spread, then a group of priori CIR characteristic and a priori error are obtained for each location after averaging 100 values.

In the identification stage, similarity between the measured CIR and the collected CIRs beforehand can be obtained with formula (16). After normalization, if the similarity between the measured CIR and that of the priori CIR under LOS condition is more than 0.7 , LOS is assumed and nothing is compensated; otherwise it is considered as NLOS. When NLOS is assumed, the compensation is equal to the ranging error caused by propagation under a single condition if the similarity between them is greater than 0.7 ; Otherwise the compensation is calculated by weighing the priori errors under various propagation conditions by formula (19). Note that we abandon the measured ranges if the similarity between the measured CIR and the priori CIRs under LOS and that when propagating around a corner conditions both are less than 0.3 .

\section{B. Results and Analysis}

The CIR characteristics of five groups of measurements under the condition of propagating around a corner are shown in table I. It can be seen that the differences between different measurements for the same characteristic is marginal. Averaging the values of five groups, the mean values of characteristic factors under five propagation conditions are shown in table II. It is clear that the differences between different propagation conditions for SNR, RT, kurtosis, skewness and RMS delay spread are similar. So we set the degree of influence of different factors as same, namely 0.2. Fig. 6 and Fig. 7 show the distribution and empirical cumulative distribution functions (CDFs) of ranging errors before and after identification and compensation. We can see that most of original ranging errors are above one meter, even up to 2.5 meters. The ranging errors after compensation are almost always below one meter, and over $90 \%$ is below 0.5 meter. Actu-

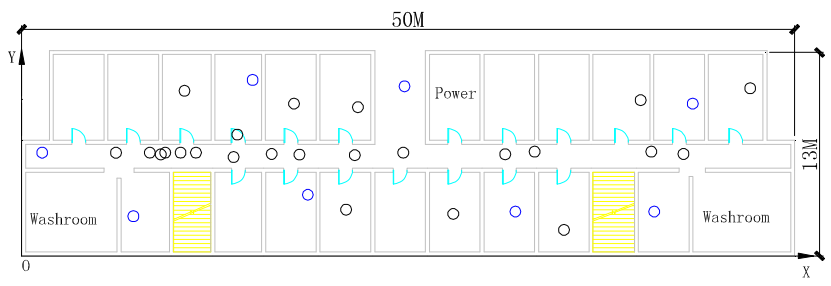

Figure. 5 Distribution of measured locations (the blue points denote the positions of responders while the black points stand for requestors). 
TABLE I. THE VALUES OF CHARACTERISTIC FACTORS IN PROPAGATING AROUND A CORNER CASES

\begin{tabular}{cccccc}
\hline & SNR & RT/ns & kurtosis & skewness & RMS delay spread/ns \\
\hline I & 27.3 & 4.2 & 209.4 & 13.2 & 4.9 \\
\hline II & 33.6 & 5.6 & 304.8 & 15.9 & 5.9 \\
\hline III & 26.6 & 5.2 & 175.3 & 11.6 & 6.6 \\
\hline IV & 36.3 & 4.0 & 309.4 & 15.5 & 4.1 \\
\hline V & 25.1 & 3.8 & 220.9 & 13.5 & 6.2 \\
\hline
\end{tabular}

TABLE II. THE VALUES OF CHARACTERISTIC FACTORS UNDER FIVE DIFFERENT PROPAGATION CONDITIONS

\begin{tabular}{cccccc}
\hline & SNR & RT/ns & kurtosis & skewness & $\begin{array}{c}\text { RMS delay } \\
\text { spread/ns }\end{array}$ \\
\hline LOS & 43.4 & 1.6 & 507.2 & 20.0 & 1.7 \\
\hline wall & 32.4 & 3.9 & 453.0 & 18.9 & 3.2 \\
\hline corner & 29.8 & 4.5 & 243.9 & 14.0 & 5.5 \\
\hline door & 45.4 & 0.7 & 844.0 & 26.5 & 1.4 \\
\hline pedestrian & 42.3 & 1.2 & 614.2 & 22.0 & 1.9 \\
\hline
\end{tabular}

ally, the number of compensated ranging errors is smaller than that of original ranging errors in Fig.6. That is because the measurements are abandoned if the similarity between the measured CIR and the priori CIRs under LOS and propagating around a corner conditions both are less than 0.3 .

Table III and Table IV show the RMSE, mean and standard deviation of measured ranges before and after compensation. The results show that the RMSE can be reduced from 0.77 to 0.33 meter, reduced by $57.1 \%$. The standard deviation of ranging errors after compensation is reduced by $54.2 \%$, implying that the difference among ranging errors is smaller. In our experiment, the success rate of identifying NLOS is not analyzed because the measurement may be considered as NLOS once the CIR is identified with abnormalities, although the measurement is performed under LOS condition and CIR is interfered by other reasons such as multipath.

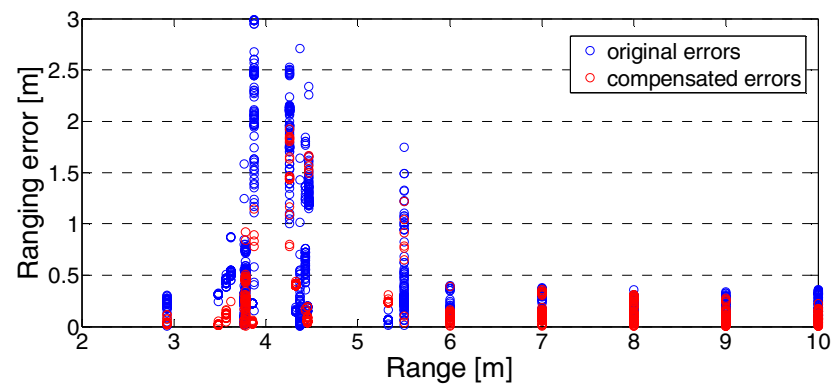

Figure 6. Ranging errors distribution of measured ranges before and after identification and compensation.

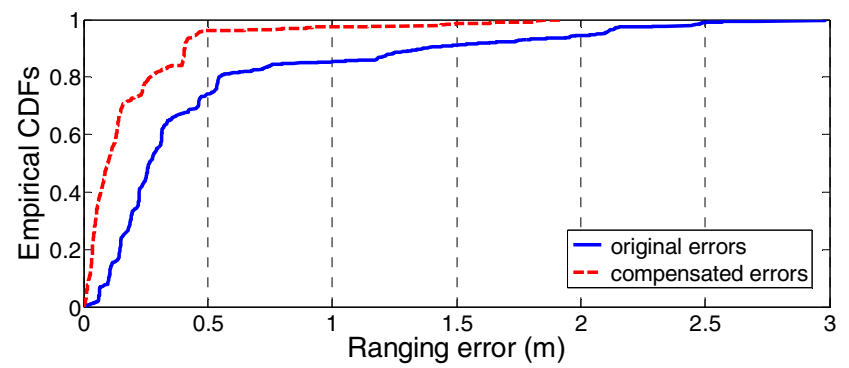

Figure 7. The empirical CDFs of ranging errors before and after identification and compensation.
TABLE III. THE RMSE OF ORIGINAL RANGES AND COMPENSATED RANGES

\begin{tabular}{ccc}
\hline & Original range & Compensated range \\
\hline RMSE $[\mathrm{m}]$ & 0.33 & 0.77 \\
\hline
\end{tabular}

TABLE IV. THE MEANS AND STANDARED DEVIATIONS OF RANGING ERRORS BEFORE AND AFTER COMPENSATION

\begin{tabular}{ccc}
\hline & $\begin{array}{c}\text { Errors before } \\
\text { compensation }\end{array}$ & $\begin{array}{c}\text { Errors after } \\
\text { compensation }\end{array}$ \\
\hline Mean $[\mathrm{m}]$ & 0.50 & 0.18 \\
\hline Standard deviation $[\mathrm{m}]$ & 0.59 & 0.27 \\
\hline
\end{tabular}

\section{CONCLUSION}

This paper proposed a method for NLOS identification and mitigation for UWB range measurements based on CIR analysis and fuzzy theory. Experimental results demonstrated the proposed method owns a good ability of identifying the quality of ranging. The ranging error below 0.5 meter is over $90 \%$ after compensation, while that before compensation is below $70 \%$, and the RMSE of measured ranges can be reduced from 0.77 to 0.33 meter. However, the capability of this method is limited to the chosen representative propagation conditions. Future work will focus on improving the compensation capability of this method and comparing the performance of this method to other state-of-the-art methods.

\section{ACKNOWLEDGEMENT}

This paper is supported by the special funding of graduate international exchange program from Wuhan University.

\section{REFERENCES}

[1] J. Khodjaev, Y. Park and A.S. Malik, "Survey of NLOS identification and error mitigation problems in UWB-based positioning algorithms for dense environments," Annals of Telecommunications. Heidelberg, vol. 65, pp. 301-311, June 2010.

[2] S. Marano, W.M. Gifford, H. Wymeersch and M.Z. Win, "NLOS identification and mitigation for localization based on UWB experimental data," IEEE Journal on Selected Areas in Communications. New Jersey, vol. 28, pp. 1026-1035, September 2011.

[3] http://www.timedomain.com/.

[4] D. Dubois and H. Prade, "Towards fuzzy differential calculus part 1: Integration of fuzzy mappings," Fuzzy Sets and Systems. Amsterdam, vol. 8, pp. 1-17, June 1982.

[ 5] H. Hashemi, "Impulse response modeling of indoor radio propagation channels," IEEE Journal on Selected Areas in Communications. New Jersey, vol. 11, pp. 594-606, September 1993.

[6] H. Hashemi and D. Tholl, "Statistical modeling and simulation of the rms delay spread of indoor radio propagation channels," IEEE Transactions on Vehicular Technology. New Jersey, vol. 43, pp. 110-120, February 1994.

[7] S. J. Howard and K. Pahlavan, "Measurement and analysis of the indoor radio channel in the frequency domain," IEEE Transactions on Instrumentation and Measurement. New Jersey, vol. 39, pp 751-755, October 1990.

[8] M. Malajner, and D. Gleich, "Soil moisture estimation using UWB," Ieee/aces International Conference on Wireless Information Technology and Systems. Hawaii, pp. 1-2, March 2016.

[9] A. Muqaibel, A. Safaai-Jazi, A. Bayram and S.M. Riad, "Ultra wideband material characterization for indoor propagation," IEEE Antennas and Propagation Society International Symposium. Lemnos, vol.4, pp. 623-626, June 2003. 